\title{
The monosynaptic Ia afferent pathway can largely explain the stretch duration effect of the long latency M2 response
}

\author{
Jasper Schuurmans · Erwin de Vlugt • \\ Alfred C. Schouten - Carel G. M. Meskers • \\ Jurriaan H. de Groot · Frans C. T. van der Helm
}

Received: 25 June 2008/Accepted: 2 November 2008/Published online: 2 December 2008

(C) The Author(s) 2008. This article is published with open access at Springerlink.com

\begin{abstract}
Sudden stretch of active muscle typically results in two characteristic electromyographic responses: the short latency M1 and the long latency M2. The M1 response originates from the monosynaptic Ia afferent reflex pathway. The M2 response is less well understood and is likely a compound response to different afferent inputs mediated by spinal and transcortical pathways. In this study the possible contribution of the Ia afferent pathway to the M2 response was investigated. A mechanism was hypothesized in which the M1 response synchronizes the motoneurons, and therewith their refractory periods. Stretch perturbation experiments were performed on the wrist and results were compared with a computational model of a pool of motoneurons receiving tonic and Ia afferent input. The simulations showed the same stretch amplitude, velocity, and duration-dependent characteristics on the M2 as found experimentally. It was concluded that the stretch duration effect of the M2 likely originates from the proposed Ia afferent mediated mechanism.
\end{abstract}

Keywords M2 response $\cdot$ Ia afferent $\cdot$ Stretch reflex

J. Schuurmans $(\bowtie) \cdot$ E. de Vlugt · A. C. Schouten .

F. C. T. van der Helm

Laboratory for Neuromuscular Control,

Department of Biomechanical Engineering,

Delft University of Technology,

Mekelweg 2, 2628 CD Delft, The Netherlands

e-mail: j.schuurmans@tudelft.nl

C. G. M. Meskers - J. H. de Groot

Department of Rehabilitation Medicine,

Leiden University Medical Center,

P.O. Box 9600, 2300 RC Leiden, The Netherlands

\section{Introduction}

When a fast stretch is applied to an active muscle, two responses are typically observed in the electromyogram (EMG) (Toft et al. 1989; Ghez and Shinoda 1978; Darton et al. 1985). The short latency M1 response, with a delay in the order of $25 \mathrm{~ms}$ for muscles in the wrist, is generally ascribed to the monosynaptic stretch reflex mediated by group Ia afferents. The origin of the long latency response M2 is still debated. With a latency in the order of $60 \mathrm{~ms}$ for the wrist, contributions of slower afferents, like group II afferents (Grey et al. 2001; Matthews 1984) and cutaneous afferents (Corden et al. 2000), have been suggested. Contributions of trans-cortical pathways to the M2 have also been found (e.g. MacKinnon et al. 2000; Lourenço et al. 2006; Capaday et al. 1991).

Task instruction plays an important role in modulation of the M2 response. Experiments where the subject was instructed to either resist the stretch perturbation or let go (Crago et al. 1976; Colebatch et al. 1979; Rothwell et al. 1980) showed that subjects could suppress EMG activity in the M2 window when instructed to let go, or enhance activity when instructed to resist. Differences in the M2 between the two tasks could easily differ by a factor of 4-10. These experiments indicated that the M2 can be modulated to functionally adapt to the task at hand. Recent findings even suggest that the $\mathrm{M} 2$ is subject to an internal model of the limb configuration, increasing functional effectiveness of the response to perturbations (Kurtzer et al. 2008).

The task dependence indicates that there is supraspinal modulation of the M2, although it does not reveal if the M2 is mediated over spinal or supraspinal pathways, or both. In a reflex study on patients with Huntington's disease who lacked an M2 in the first dorsal interosseus, the patients showed normal biceps brachii, triceps brachii, and triceps 
surae M2 responses, suggesting that the M2 response was not invariably mediated over supraspinal pathways (Thilmann et al. 1991). This indicates that trans-cortical contributions are at least not universal to all muscles and Thilmann et al. suggested that these contributions are dominant only in those muscles which receive strong direct control from the motor cortex like the fingers and the hands. In a comparison of the M2 responses in the intact, spinal, and decerebrate cat no significant differences were found (Ghez and Shinoda 1978). These results indicate that without supraspinal control the spinal pathways are sufficient to produce an M1 and M2 response.

The M2 response is a flexible reflex that can be modulated separately from the M1. During ischaemia in the flexor carpi radialis the M1 showed a more rapid and stronger decline than the M2 (Cody et al. 1987). Muscle vibration reduced the $\mathrm{M} 1$ response in the muscles of the wrist but did not significantly change the M2 (Hendrie and Lee 1978). Results for the lower limb are inconclusive. During an ischemic blockade of the lower limb the M1 was strongly decreased while the M2 remained unchanged (Grey et al. 2001), but the M1 and M2 have also been reported to be simultaneously eliminated (Fellows et al. 1993).

In summary, the M2 is likely to be a compound response where the relative importance of spinal and supraspinal contributions depends on task or activity, and also on the muscle. The focus of this study is on an aspect of the M2 that is particularly observed in the upper extremity with task instructions that result in small M2 responses (like 'let go', 'do not intervene' or 'keep a constant force'). Under these conditions stretch duration is a key factor for eliciting an M2 response (Lee and Tatton 1982; Lewis et al. 2005). For the biceps muscle, Lewis et al. found that muscle stretches with duration below a critical duration of $36 \mathrm{~ms}$ did not result in M2 responses. They suggested that the M2 in the biceps muscle is mediated by group Ia afferents and suggested three Ia-mediated mechanisms for the M2 response that could account for its stretch duration dependence: (1) decreased motoneuron firing following the M1 response (suggesting that the M2 response is an interrupted M1 response); (2) response characteristics of the muscle spindle receptors, and (3) reduced temporal summation along the reflex pathway (a certain input duration is needed for a postsynaptic neuron to reach its threshold).

This study elaborates on the suggested mechanisms of duration dependence of the M2. It is hypothesized that motoneuron pool synchronization caused by an afferent volley (Türker and Powers 1999, 2001) together with continuing Ia afferent excitation by ongoing stretch can explain the characteristics of the M2 response. The hypothesized mechanism is straightforward. When a muscle is voluntarily activated the recruited motoneurons fire tonically and asynchronously. As a response to sudden muscle stretch the muscle spindles fire a Ia afferent volley, triggering a burst of motoneuron activity: the M1 response. Pool synchronization occurs, because all recruited neurons fire a spike within a short time frame. As a result, the refractory periods of the motoneurons largely coincide. After the refractory period a second (or maybe even a third, fourth, etc.) burst of motoneuron activity may be observed if the Ia afferent input persists. This implies that the duration of the stretch needs to be long enough for an M2 and that above duration threshold the M2 depends on stretch duration.

To determine the characteristics of the M2 response, a muscle stretch experiment on the flexor carpi radialis was performed. Stretch velocity and amplitude (and therewith duration) were varied and their effects on the M1 and M2 response were determined. The proposed hypothesis was then evaluated by simulating the stretch experiments with a computational model which included modeled muscle spindles, Ia afferents, and motoneurons. The model simulations demonstrated that the Ia afferent contributes to the duration effect of the M2 response through motoneuron synchronization.

\section{Methods}

An experiment was performed to determine the effect of stretch velocity, amplitude, and duration on the short latency M1 and the medium latency M2 response in the flexor carpi radialis. Additionally, the possible contribution of the Ia afferent to the M2 response was investigated by simulating the experiment with a computer model. The model included a pool of motoneurons innervated by supra-spinal 'descending' fibers to simulate the input needed for tonic contraction and Ia afferent fibers fed by a model of the muscle spindle.

\section{Experiment}

\section{Subjects}

Nine healthy subjects (mean age $36 \pm 11$ years in the range 22-55, six women) participated in the experiment. The subjects had no history of neurological or neuromuscular disorders. Approval for the experiment was given by the Medical Ethics Committee of the Leiden University Medical Center. The subjects gave informed consent prior to the experimental procedures.

\section{Experimental procedures}

The subjects sat in a chair holding the handle of the manipulator with their dominant hand. The lower arm was restrained in an arm support such that the axis of rotation of 
Fig. 1 Experimental setup. The manipulator applied ramp-andhold trajectories on the wrist to stretch the flexor carpi radialis (FCR). The subject was asked to maintain a constant joint torque level of $1 \mathrm{Nm}$. The insert shows an example of the actual measured torque at the handle together with the torque displayed to the subject (1 Hz low-pass filtered)
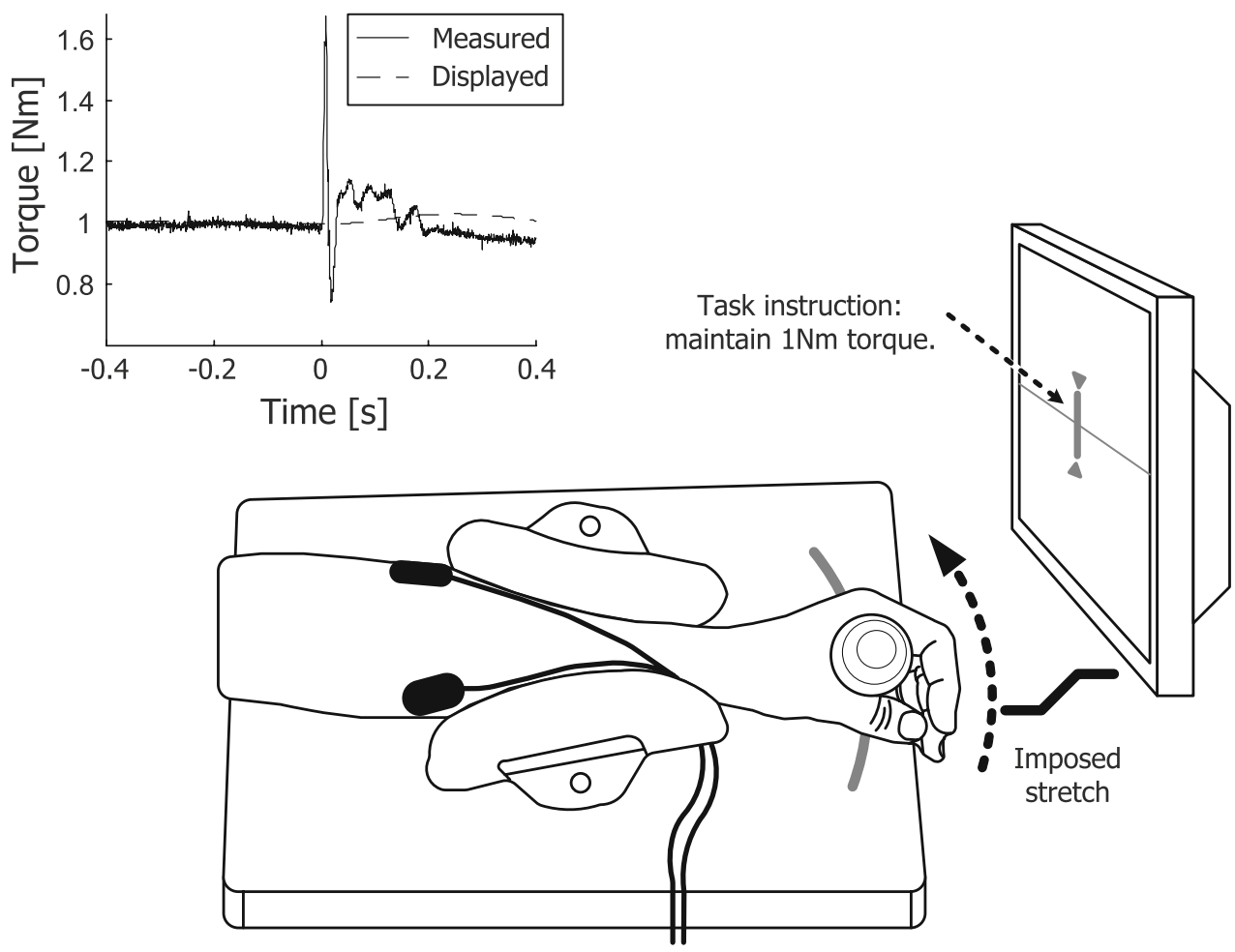

EMG the wrist and the manipulator coincided (see Fig. 1). The manipulator (Schouten et al. 2006) controlled the angle of the handle to follow ramp-and-hold trajectories. A force transducer in the handle measured the torque applied by the subject. With the wrist in its neutral position $\left(0^{\circ}\right.$ flexion) the subject was instructed to maintain a constant flexion torque of $1 \mathrm{Nm}$. This task is equivalent to a 'let go' task, in the sense that task performance is optimal when the subject gives way to the perturbations. To provide feedback the torque was low-pass filtered at $1 \mathrm{~Hz}$ and displayed on a computer screen as a moving bar. The filtering ensured that the subject did not see the rapid torque fluctuations due to the stretch perturbation acting on the inertia of the wrist, but could still perform the constant force task (see insert in Fig. 1).

A series of 12 ramp-and-hold stretch perturbations were applied, being combinations of four ramp velocities (1.5, 2.0, 3.0, and $5.0 \mathrm{rad} / \mathrm{s})$ and three ramp amplitudes (0.06, 0.10 , and $0.14 \mathrm{rad})$. All stretches were in extension direction. Each stretch perturbation was repeated 15 times, resulting in a total number of 180 perturbations. The perturbations were randomly assigned to 36 trials, containing five perturbations each. The intervals between perturbations were of randomized duration between 2.5 and $4.5 \mathrm{~s}$.

\section{Data processing}

The angle of the manipulator, the torque at the handle and the EMG of the flexor carpi radialis, and extensor carpi radialis were recorded and sampled at $2.5 \mathrm{kHz}$. The EMG was recorded with differential surface electrodes (Delsys Bagnoli system ${ }^{1}$, electrode bar length $10 \mathrm{~mm}$, bar distance $10 \mathrm{~mm}$ ). Prior to sampling the EMG signals were bandpass filtered $(20-450 \mathrm{~Hz})$.

The recorded signals (EMG, angle and torque) were separated from the original data, starting $200 \mathrm{~ms}$ prior to and ending $150 \mathrm{~ms}$ after the onset of each stretch perturbation. The separated EMG segments were rectified and low-pass filtered at $80 \mathrm{~Hz}$ (recursive third order Butterworth). Segments in which the mean flexion torque prior to onset of the perturbation deviated more than $\pm 0.1 \mathrm{Nm}$ from the instructed $1 \mathrm{Nm}$ were rejected. Each segment was separately normalized by the mean EMG prior to onset of the perturbation. The normalized segments were averaged over the 15 repetitions. After normalization, EMG values smaller than one indicate depression with respect to the background EMG and values greater than one indicate excitation.

Three metrics were derived from the rectified and normalized EMG of the flexor carpi radialis to quantify its M1 and M2 responses (Fig. 2). First, the (dimensionless) magnitude of the M1 response $A_{\mathrm{M} 1}$ was defined as the mean amplitude of the normalized EMG in the time window between 20 and $50 \mathrm{~ms}$ after stretch onset. Second, the time delay until onset of the M1 response $\left(T_{\mathrm{M} 1}\right)$ was determined as the first point in time earlier than the time of

\footnotetext{
${ }^{1}$ Delsys Inc., Boston, MA, USA.
} 

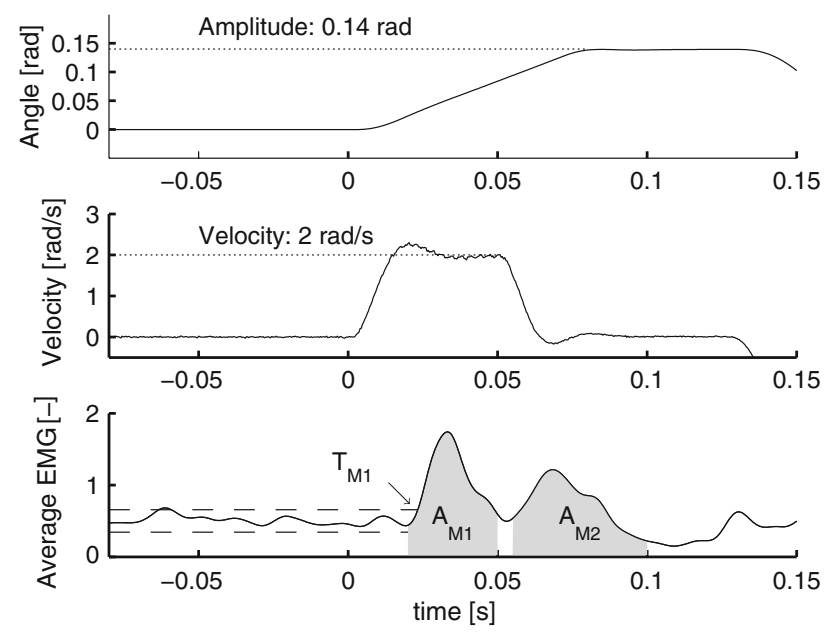

Fig. 2 Typical recording for one stretch perturbation in one subject. Stretch signal (top), stretch velocity (middle) and EMG response averaged over the 15 trials (bottom). The onset $T_{\mathrm{M} 1}$ of the M1 response was determined by the EMG exceeding three times the standard deviation of the background EMG (dotted lines show mean \pm three times SD). M1 and M2 amplitudes $A_{\mathrm{M} 1}$ and $A_{\mathrm{M} 2}$ were determined as the response area under the EMG with fixed time windows of $20-50 \mathrm{~ms}$ for the M1 and $55-100 \mathrm{~ms}$ for the M2. Areas are indicated in gray

maximum EMG, where the normalized EMG exceeded the value 1.0 by more than three times standard deviation before stretch onset. Third, the unitless magnitude of the $\mathrm{M} 2$ response $A_{\mathrm{M} 2}$ was determined as the mean value of the normalized EMG between 55 and $100 \mathrm{~ms}$ after stretch onset.

\section{Statistical analysis}

The effects of stretch velocity and amplitude on the response amplitudes $A_{\mathrm{M} 1}$ and $A_{\mathrm{M} 2}$ were tested with a two-way repeated measures analysis of variance (RM-ANOVA). The three amplitudes and four velocities combine to 12 perturbation durations. The effect of duration on the amplitude of the M2 response was tested in a one way RM-ANOVA, where duration was defined as stretch amplitude divided by stretch velocity. For all tests, a significance level of 0.05 was used.

\section{Model}

A computer model $^{2}$ (Fig. 3) was built to simulate the experiments. The model consists of a pool of 300 motoneurons. This number was chosen larger than the expected number of motoneurons recruited during the experiment, since a single simulation with a large pool was computationally more efficient than averaging multiple trials with a

\footnotetext{
${ }^{2}$ Model will be made available on http://www.3me.tudelft.nl/nmc.
}

smaller pool. The motoneurons were innervated by two sources: the tonic supraspinal input required for the constant torque, and the Ia afferent input from muscle spindles. The model output was the summation of action potentials of all 300 motoneurons, delayed by the average M1 time delay $\left(T_{\mathrm{M} 1}\right)$ found in the experiments. Like a rectified EMG signal, the model output gave a measure for the neural activity in the motoneuron pool. No additional spatiotemporal filtering was applied to simulate the electrical conduction between potentials at the muscle and the electrode pickup areas on the skin.

\section{Model inputs}

Each neuron in the population received input from 100 tonically firing descending fibers and 120 Ia afferent fibers. For the tonic input a constant rate $R_{\mathrm{T}}$ (in spikes per second) was transformed into spike trains $S_{\mathrm{T}}$; one for each fiber. This transformation was performed with a stochastic Poisson process, so each neuron received input with the same spike rate statistics, but with a different random realization.

A feline muscle spindle model (Mileusnic et al. 2006) was used to obtain the spike rate of the muscle spindles as a function of muscle stretch and stretch velocity. This muscle spindle model describes three intrafusal fibers (nuclear bag $_{1}$, and bag 2 fibers, and chain fiber) and has three inputs: normalized fascicle length $L(t)$, dynamic fusimotor activation $\gamma_{\text {dynamic }}$, and static fusimotor activation $\gamma_{\text {static }}$. This model was validated against a range of triangular, sinusoidal, and ramp-and-hold stretches.

Here, it was assumed that fascicle length scaled proportionally to whole muscle length. Normalized fascicle length $L(t)$ was obtained by converting the joint angle $\alpha$ (in radians) of the stretch perturbation to normalized muscle stretch using the muscle moment arm $r_{\text {muscle }}(12.4 \mathrm{~mm})$ and muscle length $L_{\text {muscle }}(158.5 \mathrm{~mm})$ of the flexor carpi radialis (Fridén et al. 2004):

$L(t)=1+\frac{r_{\text {muscle }}}{L_{\text {muscle }}} \cdot \alpha=1+0.08 \cdot \alpha$

The static and dynamic gamma fusimotor drives were both (arbitrarily) set at 50 spikes per second, which was within the range the spindle model was validated for. The output of the muscle spindle model was the firing rate $R_{\mathrm{Ia}}(t)$ of the Ia afferents. This firing rate was converted to individual spike trains of the fibers by a random Poisson process.

\section{Motoneuron pool}

The motoneuron pool of 300 neurons was modeled using the discrete time integrate and fire model of MacGregor 
Fig. 3 Scheme of the neural model that simulates the rampand-hold perturbation experiments. A pool of flexor motoneurons is innervated by two sources: Ia fibers that are driven by a model of the muscle spindle and tonically firing fibers. The input to this model are the ramp-and-hold stretches. The output of the model is the summation of the spikes fired by the motoneurons

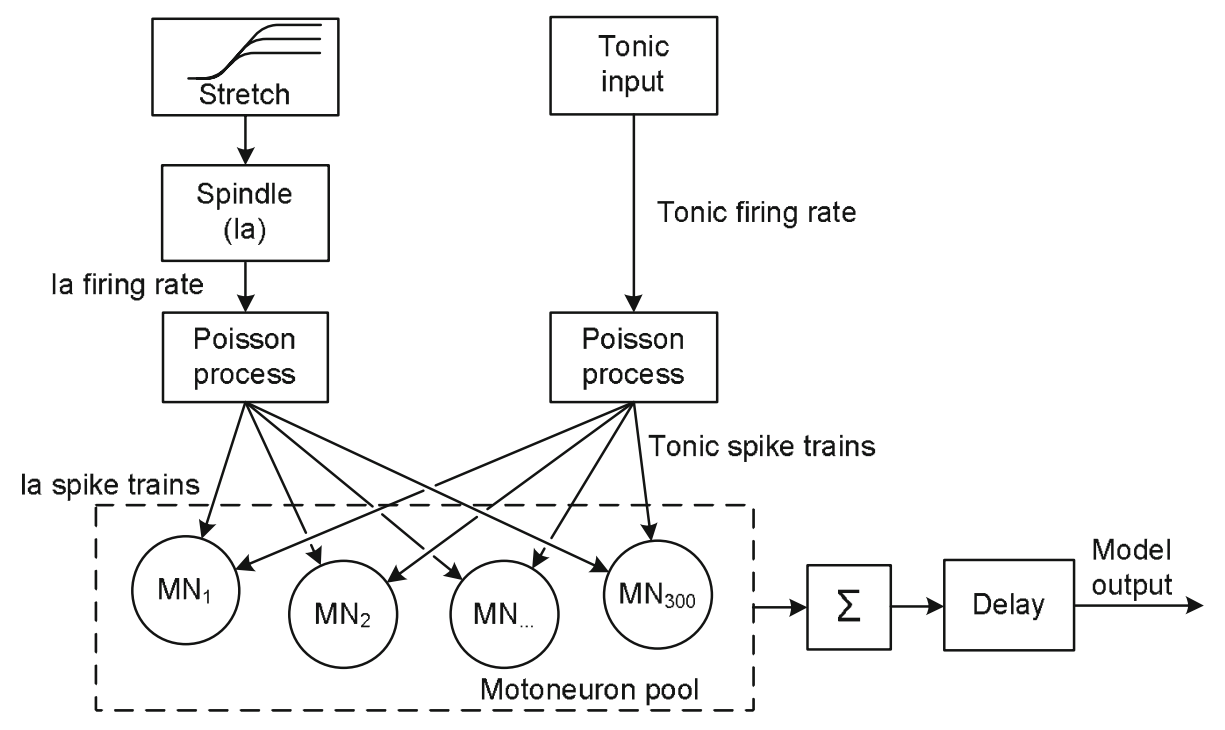

and Oliver (1974). The neuron model includes sodium and potassium conductances. Upon spike arrival of the Ia afferent and descending inputs the synaptic conductance is instantaneously increased with an amount representing the synaptic weights ( 0.01 and 0.03 , respectively). When the membrane potential reaches threshold, spike generation is modeled as a single discrete event, i.e., the action potential has no shape. The threshold of this neuron model is variable with first order dynamics and depends on the membrane potential. This captures cell accommodation. The model was adopted without modifications and was parameterized according to the motoneuron parameters in the large-scale neural model of Bashor (1998).

\section{Model simulation}

The neural model was implemented in Matlab. ${ }^{3}$ The model was run with discrete time steps of $1 \mathrm{~ms}$. To prevent transient effects of startup behavior the model was started $1 \mathrm{~s}$ prior to stretch onset. Tonic input $R_{\mathrm{T}}$ was set to 47 spikes per second to provide a background activity of the individual motoneurons of approximately 10 spikes per second $(9.8$ spikes per second was achieved) which is realistic for human motoneurons (e.g. Gorassini et al. 1998). Using this $R_{\mathrm{T}}$ the simulated EMG responses for each of the 12 stretch perturbations were determined. The simulated M1 and M2 magnitudes were determined from the motoneuron output using the same data analysis method as in the experiments. Rectification was omitted since the model output was greater than zero by definition.

\footnotetext{
3 The Mathworks, Natick, MA, USA.
}

\section{Results}

\section{Experiments}

Figure 2 shows a typical example of the averaged EMG response to one stretch perturbation of a single subject. The M1 and M2 responses (indicated in gray) are clearly visible for this combination of stretch velocity and amplitude. The M2 response is followed by a relatively silent period and a smaller third burst of activity after approximately $130 \mathrm{~ms}$. Subjects were instructed to maintain an isometric flexion torque of $1.0 \mathrm{Nm}$. A total of $5 \%$ of the stretches were rejected because the torque prior to stretch onset exceeded the $\pm 0.1 \mathrm{Nm}$ limit. The rejection percentages for individual subjects varied between 0 and $14 \%$.

Figure 4 shows the M1 and M2 responses presented as a function of stretch velocity and stretch amplitude. The M1 increased with stretch velocity $(F=17.2, P<0.001)$. Stretch amplitude had no effect on the M1 $(F=0.22$, $P=0.81$ ). The M1 response delay averaged over all subjects and all perturbations was $T_{\mathrm{M} 1}=30 \pm 5.2 \mathrm{~ms}$. The effect of stretch velocity on the delay $T_{\mathrm{M} 1}$ was remarkably high $(F=20.4, P<0.001)$. For the lowest $(1.5 \mathrm{rad} / \mathrm{s})$ and highest $(5.0 \mathrm{rad} / \mathrm{s})$ velocity the average delay times were $32.8 \pm 5.8$ and $27.8 \pm 4.7 \mathrm{~ms}$, respectively. There was no effect of stretch amplitude on the M1 delay $(F=1.3$, $P=0.31$ ).

Both stretch velocity $(F=29.7, P<0.001)$ and stretch amplitude $(F=27.7, P<0.001)$ affected the $\mathrm{M} 2$ response. There was significant interaction between velocity and amplitude $(F=5.0, P<0.001)$. The M2 increased with stretch amplitude for constant velocity and decreased with stretch velocity for constant amplitude. This suggests the correlation between the M2 response and the duration of the 
Fig. 4 Mean M1 and M2 responses as a function of stretch velocity and stretch amplitude $(n=9)$. Error bars indicate standard error of the mean. A slight offset along the horizontal axis was used to visually separate the error bars

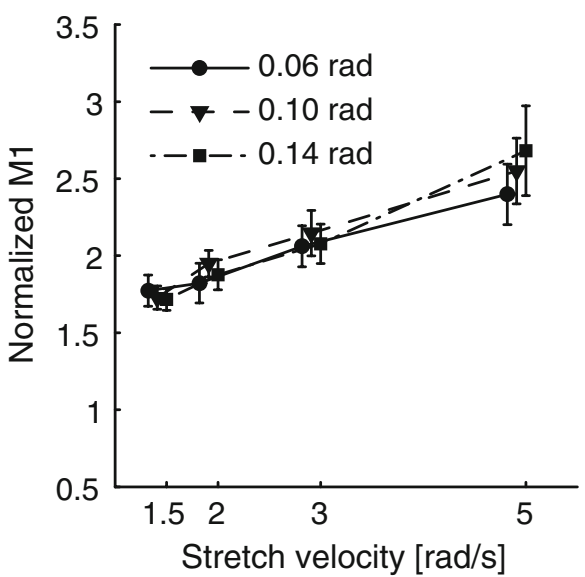

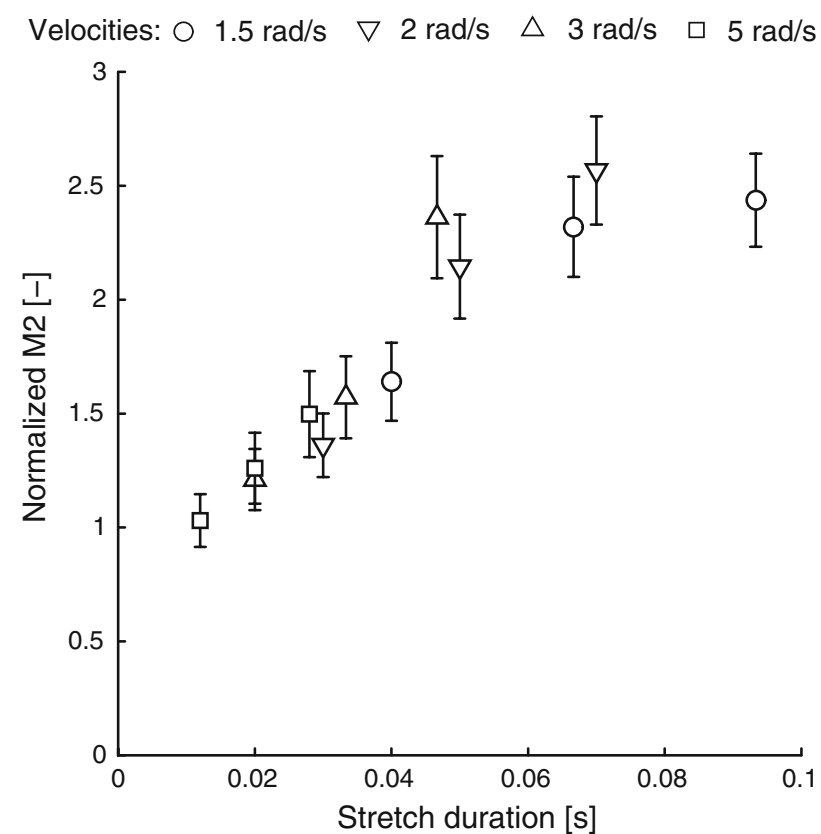

Fig. 5 Average amplitude of the M2 response as a function of perturbation duration $(n=9)$. Error bars indicate standard error of the mean

stretch, which is shown in Fig. 5. Stretch duration had a significant effect on the M2 response $(F=22.0$, $P<0.001)$. Since responses higher than 1.0 indicate excitation (and therefore the existence of an M2 response), Fig. 5 shows that perturbations shorter than approximately $30 \mathrm{~ms}$ did not trigger an M2 response. The M2 response leveled off for durations longer than 50-60 ms.

\section{Simulation results}

Figure 6 shows the output of a model simulation for one combination of stretch velocity and amplitude. The output of the motoneuron pool is comparable to the EMG recordings in Fig. 2. The motoneurons are initially firing tonically and asynchronously (Fig. 6c). The muscle stretch
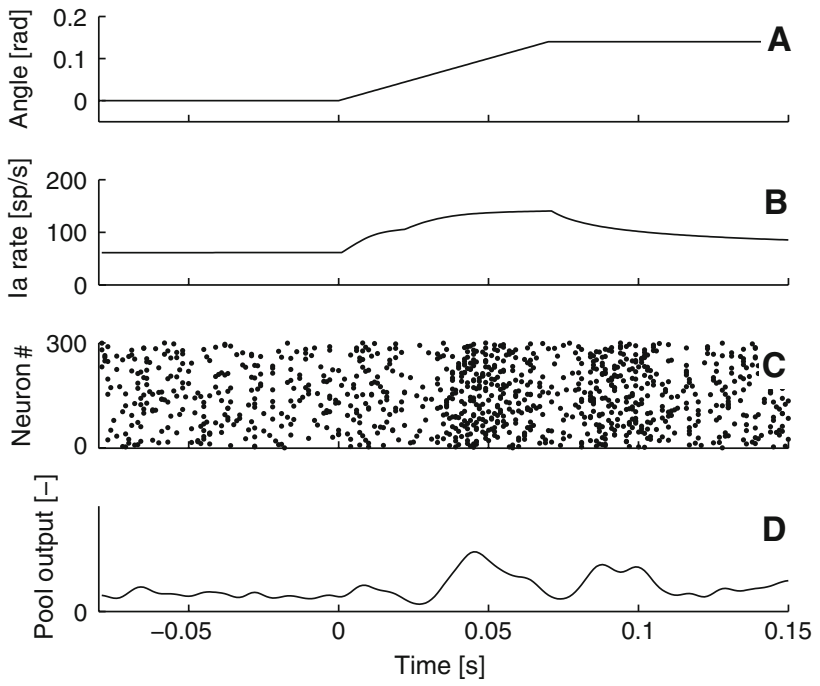

Fig. 6 Result of the model simulation for a stretch velocity of $2 \mathrm{rad} / \mathrm{s}$ and amplitude of $0.14 \mathrm{rad}$. a Joint angle. b Average firing rate of the 120 muscle spindles. c Scatter plot of the spikes fired by the 300 motoneurons. d Summed output of the motoneuron pool (compares to rectified EMG). The experimental counterpart of these model results are illustrated in Fig. 2

causes a Ia afferent volley (Fig. 6b), triggering a burst of motoneuron activity (M1). Since all motoneurons fire a spike within a short time frame synchronization occurs (dense, dark areas in Fig. 6c). As Ia afferent input continuous, a second burst of motoneuron activity occurs. As a result of this mechanism, the summed motor output (Fig. 6d) shows an M1 and M2 response. The M2 response is followed by a relatively silent period and a third burst of activity around $130 \mathrm{~ms}$ after stretch onset.

Figure 7 illustrates the effect of duration on the response of a single typical subject (A) and of the model (B). The figure shows the EMGs for three amplitudes and a constant velocity. When duration is increased by increasing amplitude, EMG activity in the M2 period increases. The model and experimental data show strong resemblance, although 
Amplitudes: $\square 0.02 \mathrm{rad} \quad \square 0.06 \mathrm{rad} \square 0.1 \mathrm{rad}$

Velocity: $1.5 \mathrm{rad} / \mathrm{s}$
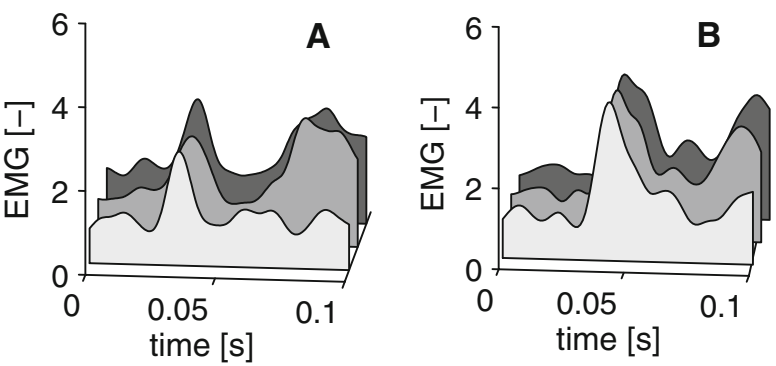

Fig. 7 Example responses of a single subject (a) and the model (b). Shades of gray indicate the three stretch amplitudes $(0.02,0.06$, $0.1 \mathrm{rad})$. Velocity is constant $(1.5 \mathrm{rad} / \mathrm{s})$

especially the M1 is wider in the model than found experimentally.

The model results on the M1 and M2 responses are illustrated in Fig. 8. Although slightly higher than that found experimentally, the simulated M1 increased with stretch velocity while stretch amplitude did not influence the M1. The simulated M2 response showed a clear decrease with increasing velocity, as did the M2 responses that were found experimentally. Only the simulated M2 responses at the combinations of highest velocity/lowest amplitude and lowest velocity/highest amplitude break this trend, although the latter was also observed experimentally. Figure 9 shows the simulated M2 as a function of stretch duration. Generally, the M2 increases linearly from nearbackground and levels off at approximately 2.2 times background for durations longer than $60 \mathrm{~ms}$. The simulated M2-duration curve has a similar shape as was found in the experimental results. The plateau level is lower $(12 \%)$ than found experimentally. Another difference between the model results and the experimental results was that the simulated M2 responses never exceeded the M1 responses, while this was the case for some of the experimental measurements.

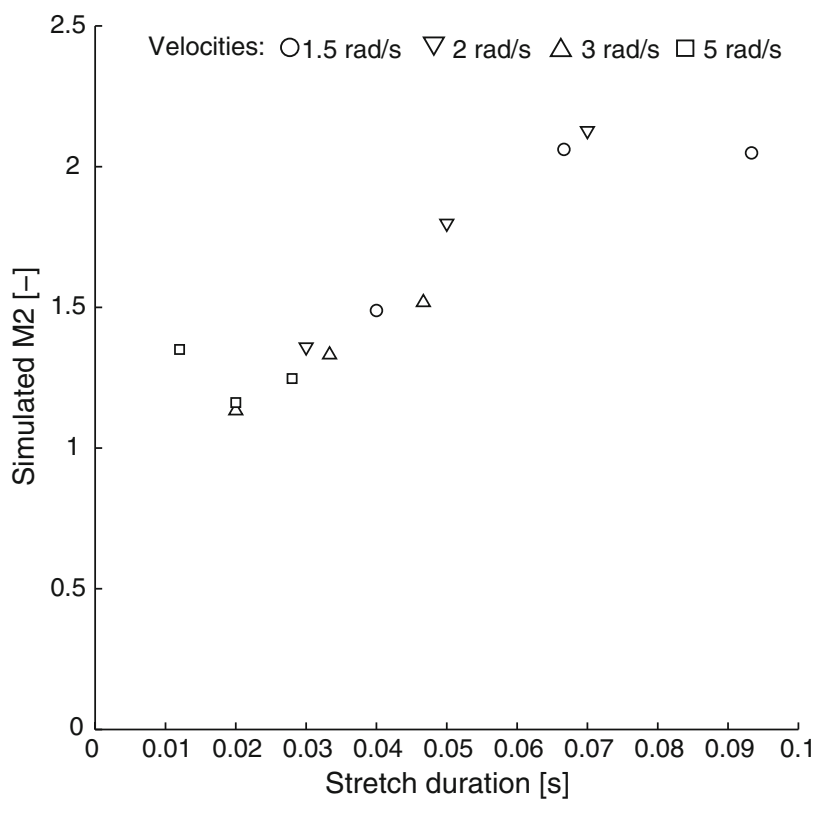

Fig. 9 Simulated M2 amplitude as a function of perturbation duration. Experimental equivalent in Fig. 5

\section{Discussion}

The goal of this study was to determine if motoneuron pool synchronization combined with ongoing Ia afferent input could explain the effects of stretch velocity, amplitude and duration on the M1 and M2 responses. To our knowledge, this is the first computational model that explains the stretch duration dependence of the M2. The muscle stretch experiment on the flexor carpi radialis confirmed earlier findings that stretch duration is the critical factor for the M2 response. Model simulations demonstrated that motoneuron synchronization triggered by a Ia afferent volley can elicit an M2 with duration characteristics that match the experimental results. Nevertheless, the proposed mechanism does not explain all aspects of the M2 response. Motoneuron synchronization alone cannot account for the
Fig. 8 Simulated M1 and M2 responses as a function of stretch velocity and stretch amplitude. Experimental equivalent in Fig. 4
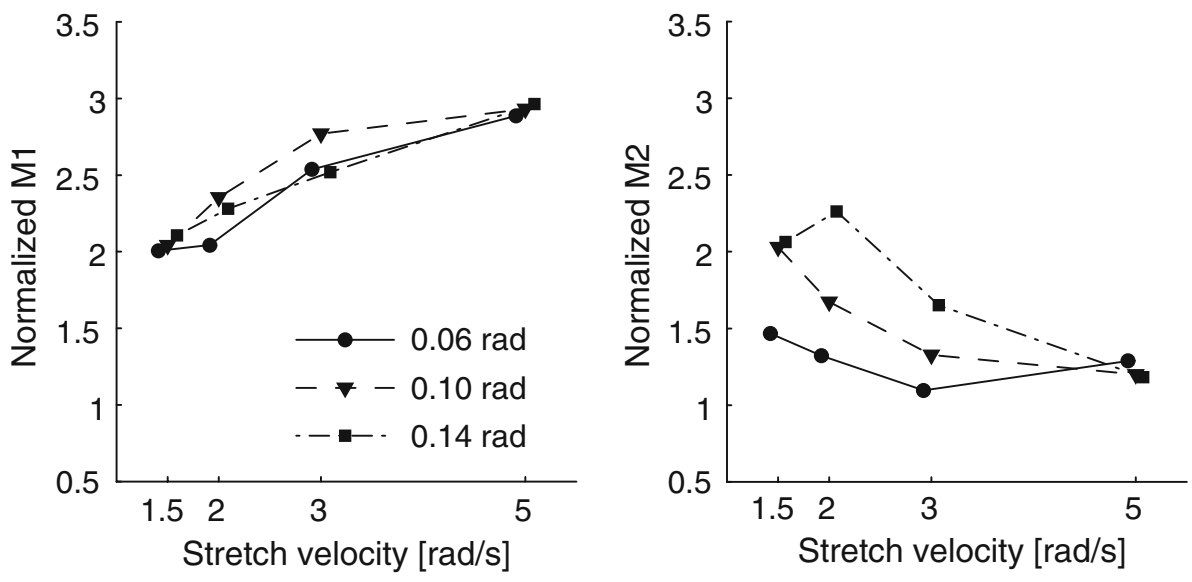
fact that M2 response can exceed the M1 response. Furthermore, the proposed mechanism does not explain differential modulation of the M1 and M2 response as reported in literature (Hendrie and Lee 1978; Cody et al. 1987). It is known that the $M 2$ is a compound response that largely depends on task instruction (Crago et al. 1976; Colebatch et al. 1979; Rothwell et al. 1980; Kurtzer et al. 2008). During 'let go'-type tasks (like the force task used here) the M2 is relatively small and the stretch duration effect is best observed. A relatively simple and generic mechanism could explain the duration effect observed in the M2 under these conditions. We conclude that the monosynaptic Ia afferent pathway is an important contributor to the duration effect observed in the M2.

\section{M1 response}

The M1 response increased with stretch velocity. Stretch velocity directly increases the output of the muscle spindles, and therewith the input to the motoneurons. Stretch amplitude had no effect on the M1; the short latency M1 is triggered while the stretch is still ongoing and the final amplitude of the stretch is not yet reached. The time delay to M1 onset decreased significantly with stretch velocity. The difference between the fastest and slowest stretch was $4.8 \mathrm{~ms}$, which was of such magnitude that it could not be explained by an artifact in the acceleration of the position controlled motor. This finding most likely demonstrates the integration of inputs of the motoneurons. The time for the motoneurons to reach their threshold decreases when the Ia afferent input increases.

The simulations showed an M1 that increased with velocity. The simulated M1 was somewhat higher than that found experimentally; this is likely caused by the homogeneity of the delay times of the afferent fibers and of the modeled motoneuron pool. All muscle spindle input arrives simultaneously at the motoneurons and all motoneurons have the same threshold. As soon as the Ia afferent input is sufficient to trigger the M1 response, all motoneurons in the pool can contribute to this response. Including variability in the time delays and motor unit recruitment would decrease the amplitude of the simulated M1.

\section{M2 response}

Depending on stretch velocity and amplitude the subjects and the computer simulations both showed clear M2 responses. The strong duration effect in the M2 confirmed the findings of Lee and Tatton (1982) at the flexor carpi radialis and Lewis et al. (2005) at the biceps, for a wide range of combinations of stretch velocities and amplitudes. The curve of M2 magnitude as a function of duration (Fig. 5) showed that for durations below $20 \mathrm{~ms}$ no M2 was triggered (EMG equal to background). For longer durations the M2 increased until it saturated at around $50 \mathrm{~ms}$. Lee and Tatton (1982) found a threshold duration of $43 \mathrm{~ms}$, while $20 \mathrm{~ms}$ was found in this study. The difference may be caused by the different joint torques used in the experiments. Lee and Tatton used $0.5 \mathrm{Nm}$, this study $1 \mathrm{Nm}$. Muscle spindle sensitivity increases with torque, and M1 and M2 responses are known to scale with contraction level (Toft et al. 1989). As in the experiments, duration was the main factor in the simulated M2 responses. The curve of simulated M2 against stretch duration strongly resembled the experiments.

The tonic drive to the motoneurons $\left(R_{\mathrm{T}}\right)$ and the static and dynamic drive to the muscle spindles $\left(\gamma_{\mathrm{s}}\right.$ and $\left.\gamma_{\mathrm{d}}\right)$ were arbitrarily chosen within plausible biological bounds. To determine the robustness of the proposed M2 mechanism against variations in these parameters a sensitivity analysis was performed. The tonic drive to the motoneurons $R_{\mathrm{T}}$ and gamma drive $\gamma_{\mathrm{s}}$, and $\gamma_{\mathrm{d}}$ were individually increased and decreased with an amount such that the net motoneuron firing frequency changed with $\pm 25 \%$. The main effect observed in the M2 responses was that the M2 increased with respect to background activity when motoneuron frequency decreased and vice versa. The maximum absolute change in the average M2 with respect to nominal values was an increase of $13 \%$ when motoneuron frequency was decreased by $25 \%$. No change was observed in the trend that the M2 increased with duration and decreased with velocity.

Our hypothesis was that the burst of Ia afferent input after muscle stretch synchronizes the motoneuron pool by triggering an action potential in many neurons within a short time frame. This initial burst is called the M1 response. According to the hypothesis an M2 response would be observed when the excitation by Ia afferents lasts long enough for the synchronized neurons to fire a second spike. The simulation response in Fig. 6c clearly demonstrated the hypothesized mechanism. According to the hypothesis, a third burst of activity might just as well appear. The subject response in Fig. 2 showed a third burst of activity after approximately $130 \mathrm{~ms}$, which was typical to other subjects as well and was also observed in the computer simulations in Fig. 6. However, it cannot be excluded that these responses in humans were due to voluntary activation, given the long latencies.

Both in the experiments and the simulations the M2 response increased with amplitude when velocity was constant and decreased with stretch velocity when amplitude was constant. These findings indicate that at least the II afferent could not have been the main contributor to the M2. Higher stretch velocities decrease the time to reach the plateau amplitude. Since the II afferent encodes muscle length, higher velocities would make the II afferent reach its maximal activity earlier. As a result, the 
motoneurons would receive a larger amount of net afferent input. Since the M2 decreased instead of increased with velocity, the II afferent is unlikely to be the main contributor to the M2.

The proposed M2 mechanism explains the mean subject behavior in the experimental finding well, but does not explain that the M2 response can exceed the M1 response. In this experiment this only occurred in some subjects for stretches with long duration, but literature reports on consistent higher M2's than M1's for other experimental conditions (e.g. Kurtzer et al. 2008). This finding is concordant with the notion that the M2 probably is a compound response in which more than one (spinal or trans-cortical) pathway plays a role.

Although not evoked by the current experimental protocol, differential modulation of the M1 and M2 observed in other protocols cannot be explained by the proposed mechanism. We therefore argue that the presented model explains the duration dependence of the M2, which is typically observed in protocols inducing relatively small M2 responses, but not the modulation effects observed in protocols which induce 'large' M2 responses.

\section{Model constraints}

The straightforward model presented here was capable to demonstrate the velocity, amplitude and duration characteristics of the M1 and M2. With refraction and synchronization being such basic neural phenomena, more sophisticated models (requiring more assumptions) are unlikely to affect the main findings. The neuronal model describes the dynamics of the membrane potential, neglecting the fast dynamics of the actual action potential generation. For our synchronization hypothesis, however, this suffices: the two key mechanisms in our hypothesis (i.e. firing a spike that synchronizes the motoneuron pool and the refractory period after a spike was fired) are both covered by the model. Parameter values were assumed to be homogeneous for all motoneurons in the pool, as no data are available on the distribution in vivo. Introducing spread in the parameter values would cause decreasing synchronicity leading to widening and decreasing M1 and M2 peak amplitudes. Still, if the afferent input is large enough to trigger an M1 response, the pool will be synchronized by simultaneously firing a spike. We used the muscle spindle model of Mileusnic et al. (2006). Alternative models would probably not affect our results, since the main aspect of the afferent input is its sensitivity to stretch velocity that triggers the $\mathrm{M} 1$ and $\mathrm{M} 2$ responses.

Acknowledgments The authors would like to thank Wendy de Graaf for her contributions to the experiments. This study was performed within trauma related neuronal dysfunction (TREND), a knowledge consortium that integrates research on complex regional pain syndrome type 1 . The project is supported by a Dutch Government grant (BSIK03016).

Open Access This article is distributed under the terms of the Creative Commons Attribution Noncommercial License which permits any noncommercial use, distribution, and reproduction in any medium, provided the original author(s) and source are credited.

\section{References}

Bashor DP (1998) A large-scale model of some spinal reflex circuits. Biol Cybern 78:147-157

Capaday C, Forget R, Fraser R, Lamarre Y (1991) Evidence for a contribution of the motor cortex to the long-latency stretch reflex of the human thumb. J Physiol 440:243-255

Cody FWJ, Goodwin CN, Richardson HC (1987) Effects of ischaemia upon reflex electromyographic responses evoked by stretch and vibration in human wrist flexor muscles. J Physiol 391:589-609

Colebatch JG, Gandevia SC, McCloskey DI, Potter EK (1979) Subject instruction and long latency reflex responses to muscle stretch. J Physiol 292:527-534

Crago PE, Houk JC, Hasan Z (1976) Regulatory actions of human stretch reflex. J Neurophysiol 39(5):925-935

Corden DM, Lippold OC, Buchanan K, Norrington C (2000) Longlatency component of the stretch reflex in human muscle is not mediated by intramuscular stretch receptors. J Neurophysiol 84(1):184-188

Darton K, Lippold OC, Shahani M, Shahani U (1985) Long-latency spinal reflexes in humans. J Neurophysiol 53(6):1604-1618

Fellows SJ, Dömges F, Töpper R, Thilmann AF, Noth J (1993) Changes in the short- and long-latency stretch reflex components of the triceps surae muscle during ischaemia in man. J Physiol 472:737-748

Fridén J, Lovering RM, Lieber RL (2004) Fiber length variability within the flexor carpi ulnaris and flexor carpi radialis muscles: implications for surgical tendon transfer. J Hand Surg 29A:909914

Ghez C, Shinoda Y (1978) Spinal mechanisms of the functional stretch reflex. Exp Brain Res 32(1):55-68

Gorassini MA, Bennetta DJ, Yang JF (1998) Self-sustained firing in human motor units. Neurosci Lett 247(1):13-16

Grey MJ, Ladouceur M, Andersen JB, Nielsen JB, Sinkjaer T (2001) Group ii muscle afferents probably contribute to the medium latency soleus stretch reflex during walking in humans. J Physiol 534(3):925-933

Hendrie A, Lee RG (1978) Selective effects of vibration on human spinal and long-loop reflexes. Brain Res 157:369-375

Kurtzer IL, Pruszynski JA, Scott SH (2008) Long-latency reflexes of the human arm reflect an internal model of limb dynamics. Curr Biol 18:449-453

Lee RG, Tatton WG (1982) Long latency reflexes to imposed displacements of the human wrist: dependence on duration of movement. Exp Brain Res 45(1-2):207-216

Lewis GN, Perreault EJ, MacKinnon CD (2005) The influence of perturbation duration and velocity on the long-latency response to stretch in the biceps muscle. Exp Brain Res 163(3):361-369

Lourenço G, Iglesias C, Cavallari P, Pierrot-Deseilligny E, MarchandPauvert V (2006) Mediation of late excitation from human hand muscles via parallel group II spinal and group I cortical pathways. J Physiol 572.2:585-603

MacGregor RJ, Oliver RM (1974) A model for repetitive firing in neurons. Kybernetik 16(1):53-64 
MacKinnon CD, Verrier MC, Tatton WG (2000) Motor cortical potentials precede long-latency EMG activity evoked by imposed displacements of the human wrist. Exp Brain Res 131(4):477-490

Matthews PBC (1984) Evidence from the use of vibration that the human long-latency stretch reflex depends upon spindle secondary afferents. J Physiol 348:383-415

Mileusnic MP, Brown IE, Lan N, Loeb GE (2006) Mathematical models of proprioceptors. I. Control and transduction in the muscle spindle. J Neurophysiol 96:1772-1788

Rothwell JC, Traub MM, Marsden CD (1980) Influence of voluntary intent on the human long-latency stretch reflex. Nature 286:496498

Schouten AC, de Vlugt E, van Hilten JJ, van der Helm FC (2006) Design of a torque-controlled manipulator to analyse the admittance of the wrist joint. J Neurosci Methods 154(12):134-141
Thilmann AF, Schwarz M, Töpper R, Fellows SJ, Noth J (1991) Different mechanisms underlie the longlatency stretch reflex response of active human muscle at different joints. J Physiol 444:631-643

Toft E, Sinkjaer T, Andreassen S (1989) Mechanical and electromyographic responses to stretch of the human anterior tibial muscle at different levels of contraction. Exp Brain Res 74(1):213-219

Türker KS, Powers RK (1999) Effects of large excitatory and inhibitory inputs on motoneuron discharge rate and probability. J Neurophysiol 82:829-840

Türker KS, Powers RK (2001) Effects of common excitatory and inhibitory inputs on motoneuron synchronization. J Neurophysiol 86:2807-2822 